XXXI International Workshop on High Energy Physics:

Critical Points in the Modern Particle Physics

International Journal of Modern Physics: Conference Series

Vol. 47 (2018) 1860101 (7 pages)

(C) The Author(s)

DOI: $10.1142 / \mathrm{S} 2010194518601011$

\title{
Quartet-Metric Gravity and Dark Components of the Universe
}

\author{
Y. F. Pirogov \\ SRC Institute for High Energy Physics of NRC Kurchatov Institute, \\ Protvino, 142281 Moscow Region, Russia \\ pirogov@ihep.ru
}

Published 6 June 2018

\begin{abstract}
In the report there are presented the general frameworks for the quartet-metric gravity based on the two main principles. First, there exist in space-time the distinct dynamical coordinates, given by a scalar quartet, playing the role of the Higgs fields for gravity. Second, the physical gravity fields arising due to the spontaneous symmetry breaking serve as the dark components of the Universe. It is argued that the mere admixture to metric of the scalar quartet may give rise to an extremely wide spectrum of the emergent gravity phenomena beyond General Relativity (GR). Further developing the proposed frameworks to find out the next-to-GR theory of gravity is a challenge.
\end{abstract}

Keywords: Modified gravity; spontaneous symmetry breaking; dark matter; dark energy.

\section{Introduction: dark components and quartet-metric gravity}

The current state-of-the-art in cosmology may be summarized by the so-called Standard Cosmological Model (or, otherwise, the $\Lambda \mathrm{CDM}$ model). ${ }^{\mathrm{a}}$ According to the model, the Universe expands with acceleration, is spatially flat $(K=0)$ with high precision, and possesses at the present epoch by the following

- Energy content: $\Omega_{K}=0, \Omega_{r}+\Omega_{b}+\Omega_{D M}+\Omega_{\Lambda}=1$, with the partial contributions:

(a) Radiation negligibly small $\Omega_{r} \ll 1$;

(b) Baryonic matter $\Omega_{b} \simeq 0.04$;

(c) Cold dark matter $(\mathrm{CDM}) \Omega_{D M} \simeq 0.26$ (the total matter $\Omega_{b+D M} \simeq 0.3$ );

(d) Vacuum energy $/ \Lambda$-term $\Omega_{\Lambda} \simeq 0.7$.

The $\Lambda$ CDM model proves to be highly successful phenomenologically revealing nevertheless some (mainly theoretical)

\footnotetext{
${ }^{\text {a See, e.g. Ref. } 1 .}$
}

This is an Open Access article published by World Scientific Publishing Company. It is distributed under the terms of the Creative Commons Attribution 4.0 (CC-BY) License. Further distribution of this work is permitted, provided the original work is properly cited. 


\section{- Problems:}

(a) Nature and properties of DM;

(b) Cosmological constant (CC) $/ \Lambda$-term (un)naturalness: why $\Lambda$ is extremely small compared to the Planck mass $M_{P l}$ (or even the electroweak scale $\left.M_{W}\right)$ ?

The solution to the problems may imply, among other things, going beyond General Relativity (GR), on which $\Lambda \mathrm{CDM}$ is based. In the absence of the convincing observational and/or theoretical arguments in favor of a preferred choice for the beyond GR theory of gravity (if any), it it is natural to try and modify GR restricting himself exclusively by the gravity components already present in metric. ${ }^{\mathrm{b}}$ In GR, the metric components beyond the tensor graviton get non-physical due to the general relativity, or, technically, the general diffeomorphism (GDiff) invariance. To lift these gauge components from the gauge "cellar" to the observational "attic", converting them into the physical dark components (DCs), the mechanism of the spontaneous symmetry breaking (SSB) for gravity is to be implemented. To this end, in Ref. ${ }^{2}$ there were developed the frameworks of the spontaneously broken GR, with the distinct dynamical coordinates, given by a scalar quartet, playing the role of the Higgs-like fields for gravity. The so-constructed quartet-metric gravity (QMG) describes, in general, the scalar-vector-tensor gravity (or some of its reductions). At that, the physical gravity components arising due to SSB are to be treated as the gravitational DM and/or DE, depending on the context. ${ }^{3,4}$ The respective frameworks, with some elaboration, are exposed in what follows.

\section{Quartet-metric gravity: general frameworks}

\subsection{Basic ingredients}

Whatever the underlying theory of gravity might be, to be eventually confronted with Particle Standard Model, as well as the observations, it should be realized on the present day level (or even the level of a foreseeable future) as the effective field theory (EFT). The latter is generically characterized by the three basic ingredients: the set of fields, the pattern of symmetries and the type of interactions among the given fields satisfying the given symmetries. QMG may be characterized as follows.

- Field set:

(a) Gravity field/metric $g_{\mu \nu}(x)$;

(b) Scalar quartet $Z^{a}(x), \quad a=0, \ldots 3$, defining on space-time the distinct (chartvise reversible) dynamical coordinates $z^{\alpha}=\delta_{a}^{\alpha} Z^{a}(x), x^{\mu}=x^{\mu}(z)$, with $x^{\mu}$ the arbitrary observer's coordinates. By default, such distinct quasi-affine coordinates $z^{\alpha}$ are attributed to the (chart-wise homogeneous) vacuum.

\section{- Symmetry pattern:}

\footnotetext{
bIn this respect, one may invoke the principle of the so-called Occam's razor: "Among the competing hypotheses, the one(s) with the fewest assumptions should be preferred".
} 
(a) General covariance (GC): all the (smooth enough) coordinates $x^{\mu}$ (including, in particular, $\left.z^{\alpha}\right)$ on the space-time manifold are a priori admissible on par. ${ }^{\mathrm{c}}$

(b) General relativity (GR)/general diffeomorphism (GDiff) invariance under

$$
\text { GDiff }: x^{\mu} \rightarrow x^{\mu}-\xi^{\mu}(x), \quad\left|\xi^{\mu}\right| \ll 1,
$$

followed by the associated transformation of the matter fields through their Lie derivative. At that, GDiff serves as the gauge group for gravity.

(c) Poincare invariance:

$$
I S O(1,3): Z^{a} \rightarrow \Lambda_{b}^{a} Z^{b}+c^{a},
$$

with the (chart-wise) global translations $c^{a}$ and Lorentz transformations $\Lambda$ living invariant the Minkowski symbol $\eta_{a b}$.

- Type of interactions: defined generically by the gauge and Lorentz-invariant GC scalar action

$$
S=\int \mathcal{L}\left(g_{\mu \nu}, \partial_{\lambda} g_{\mu \nu}, Z_{\mu}^{a}, \partial_{\mu} Z_{\nu}^{a}, \Psi, \partial_{\mu} \Psi, \eta_{a b}\right) d^{4} x
$$

with $\mathcal{L}$ the Lagrangian density in terms of the tetrad $Z_{\mu}^{a} \equiv \partial_{\mu} Z^{a}$ and a generic matter field $\Psi$. $^{\mathrm{d}}$

\subsection{Basic constructions}

The looked-for GC scalar action may straightforwardly be constructed in terms of the GC elements as follows.

- Connection-like tensor: ${ }^{2}$

$$
B_{\mu \nu}^{\lambda}(x) \equiv \Gamma_{\mu \nu}^{\lambda}\left(g_{\mu \nu}\right)-\partial_{\mu} \partial_{\nu} z^{\alpha} \partial x^{\lambda} /\left.\partial z^{\alpha}\right|_{z=Z(x)},
$$

where $z^{\alpha} \equiv \delta_{a}^{\alpha} Z^{a}(x)$ and, inversely, $x^{\lambda}=x^{\lambda}(z)$. At that, in the quasi-affine coordinates $z^{\alpha}$ one gets $B_{\alpha \beta}^{\gamma}(z)=\Gamma_{\alpha \beta}^{\gamma}\left(g_{\alpha \beta}\right)$.

- Quasi-affine metric:

$$
\zeta_{\mu \nu}=Z_{\mu}^{a} Z_{\nu}^{b} \eta_{a b}
$$

in terms of which one gets, in particular,

$$
B_{\mu \nu}^{\lambda}=\Gamma_{\mu \nu}^{\lambda}\left(g_{\mu \nu}\right)-\gamma_{\mu \nu}^{\lambda}\left(\zeta_{\mu \nu}\right)
$$

as the difference of the two Christoffel connections. ${ }^{\text {e }}$

- Scalar-graviton:

$$
\sigma \equiv 1 / 2 \ln g / \zeta
$$

\footnotetext{
${ }^{\mathrm{c}}$ Paraphrasing the well-known maxim one may thus say that "all coordinates are equivalent but some are more equivalent than others".

dDue to the assumed (chart-wise) global Poincare invariance, reflecting the homogeneity of the vacuum, there is no derivativeless input of $Z^{a}$. This allows to treat the second derivative of $Z^{a}$ just as the first derivative of the tetrad $Z_{\mu}^{a}$.

${ }^{\mathrm{e}}$ It follows that in the quasi-affine coordinates $z^{\alpha}$ one has $\zeta_{\alpha \beta}(z)=\eta_{\alpha \beta}, \zeta=-1$ and $\gamma_{\alpha \beta}^{\gamma}(z)=0$, what, in fact, distinguishes such coordinates.
} 
composed of the two scalar densities $g \equiv \operatorname{det}\left(g_{\mu \nu}\right)$ and $\zeta \equiv \operatorname{det}\left(\zeta_{\mu \nu}\right)$.

- Higgs-like scalar: ${ }^{\mathrm{f}}$

$$
\Sigma^{a b} \equiv g^{\kappa \lambda} Z_{\kappa}^{a} Z_{\lambda}^{b}
$$

or, equivalently, the Higgs-like tensor

$$
\Sigma^{\mu}{ }_{\nu} \equiv Z_{a}^{\mu} \Sigma^{a c} \eta_{c b} Z_{\nu}^{b}=g^{\mu \lambda} \zeta_{\lambda \nu}
$$

with $Z_{a}^{\mu} \equiv \partial x^{\mu} /\left.\partial z^{\alpha}\right|_{z=Z(x)}$ the tetrad inverse to $Z_{\mu}^{a}$. This implies the relation

$$
\operatorname{det}\left(\Sigma^{a c} \eta_{c b}\right)=\operatorname{det}\left(\Sigma^{\mu}{ }_{\nu}\right)=\zeta / g=e^{-2 \sigma}
$$

and defines another scalar composed of the two basic metrics:

$$
s_{1} \equiv \Sigma^{a b} \eta_{b a}=\Sigma_{\mu}^{\mu}=g^{\mu \nu} \zeta_{\nu \mu},
$$

as well as similar constructions of the higher degrees, like $s_{2}=\Sigma^{\mu}{ }_{\nu} \Sigma^{\nu}{ }_{\mu} \neq s_{1}^{2}$, etc.

- Space-time measure $\mathcal{M}$ for the volume element $d V \equiv \mathcal{M} d^{4} x$ :

(a) Gravitational: $\mathcal{M}_{g}=\sqrt{-\operatorname{det}\left(g_{\mu \nu}\right)} \equiv \sqrt{-g}$;

(b) Quasi-affine/non-gravitating: ${ }^{\mathrm{g}} \mathcal{M}_{\zeta}=\operatorname{det}\left(\partial_{\mu} Z^{a}\right)=\sqrt{-\operatorname{det}\left(\zeta_{\mu \nu}\right)} \equiv \sqrt{-\zeta}$.

All the constructions are understood ultimately as the functions of the basic fields $g_{\mu \nu}$ and $Z_{\mu}^{a}=\partial_{\mu} Z^{a}$. In building the action, one should, in particular, account for

- Measure/Lagrangian ambiguity: without loss of generality one can always choose either the gravitational or quasi-affine measure (or a combination of both) supplemented by a proper redefinition of the Lagrangian, which one gets simpler.

Imposing on space-time the geometrical structure one gets more elaborate elements.

- Quartet-modified metric:

$$
\tilde{g}_{\mu \nu}=f(\Sigma) g_{\mu \nu}+\varphi(\Sigma) \zeta_{\mu \nu}
$$

as a linear combination of the two basic metrics, with the $\Sigma$-dependent coefficients. This defines, in turn, the quartet-modified Higgs-like tensor

$$
\tilde{\Sigma}^{\mu} \nu \equiv \tilde{g}^{\mu \lambda} \zeta_{\lambda \nu}=f(\Sigma) \Sigma^{\mu}{ }_{\nu}+\varphi(\Sigma) \delta_{\nu}^{\mu},
$$

the connection-like tensor

$$
\tilde{B}_{\mu \nu}^{\lambda} \equiv \Gamma_{\mu \nu}^{\lambda}\left(\tilde{g}_{\mu \nu}\right)-\gamma_{\mu \nu}^{\lambda}\left(\zeta_{\mu \nu}\right)
$$

and the measure

$$
\tilde{\mathcal{M}}=\sqrt{-\operatorname{det}\left(\tilde{g}_{\mu \nu}\right)} \equiv \sqrt{-\tilde{g}}
$$

A priori, it is conceivable using in the action $S$ the different metrics. To reduce such an ambiguity one may additionally impose

${ }^{\mathrm{f}}$ Such a scalar to implement the Higgs mechanism for graviton was proposed originally in Refs. ${ }^{5-7}$ $\mathrm{g}$ The quartet of scalar fields for the dynamical non-gravitating measure was proposed originally in Ref. ${ }^{8}$ For a subsequent elaboration, see, e.g. ${ }^{9}$ 
- Geometry universality: all the geometrical constructions on the space-time, such as the Christoffel connection $\tilde{\Gamma}_{\mu \nu}^{\lambda}$, the covariant derivative $\tilde{\nabla}_{\mu}$, the measure $\tilde{\mathcal{M}}=\sqrt{-\tilde{g}}$, etc, are to be attributed to the single metric $\tilde{g}_{\mu \nu}$. At $\tilde{g}_{\mu \nu} \neq g_{\mu \nu}$ the geometry/gravity equivalence is violated due to influence of the vacuum on metric.

Under the chosen geometry, the residual freedom in the action $S$ is to be attributed to the choice of the Lagrangian $L$ specifying the dynamics. By this token one gets

- GC scalar action

$$
S=\int L\left(\tilde{R}, \tilde{B}_{\mu \nu}^{\lambda}, \tilde{g}_{\mu \nu}, \tilde{\Sigma}^{\mu}{ }_{\nu}, \tilde{\nabla}_{\mu} \Psi, \Psi\right) \tilde{\mathcal{M}} d^{4} x
$$

with $\tilde{R} \equiv R\left(\tilde{g}_{\mu \nu}\right)$ the Ricci scalar, and the Lagrangian $L=K-V+L_{m}$ containing

(a) Kinetic term: $K\left(\tilde{R}, \tilde{B}_{\mu \nu}^{\lambda}, \tilde{g}_{\mu \nu}, \tilde{\Sigma}\right)$;

(b) Potential: $V(\tilde{\Sigma})$;

(c) Matter Lagrangian: $L_{m}\left(\tilde{\nabla}_{\mu} \Psi, \Psi, \tilde{g}_{\mu \nu}, \tilde{\Sigma}\right)$.

Admitting in $L$ conventionally not higher then the first degree of derivative of $g_{\mu \nu}$ and $Z_{\mu}^{a}$ one may, following the simplicity spirit, impose on $S$ additionally the reduction of the two following principle types.

- Quadratic-derivative: leaving not higher than the second power of the first derivative of $g_{\mu \nu}$ and $Z_{\mu}^{a}$, but a priori any input of $\zeta_{\mu \nu}$ (including those through $\zeta$ );

- Scalar-graviton: leaving the input of $\zeta_{\mu \nu}$ only through its determinant $\zeta$, but a priori any power of the first derivative of $\sigma$.

Imposing both types of reduction for the scalar graviton results in the maximal simplification (see, below).

\section{Quartet-modified GR: quadratic-derivative reduction}

\subsection{Non-linear theory}

To illustrate the general frameworks let us consider in more detail the quartetmodified GR, ${ }^{2}$ which still possesses by the geometry/gravity equivalence as GR, $\tilde{g}_{\mu \nu}=g_{\mu \nu}$ and $\mathcal{M}=\sqrt{-g}$, though with the modified quadratic-derivative action:

$$
S=\int\left(L_{g}\left(g_{\mu \nu}\right)+\Delta L_{g}\left(g_{\mu \nu}, \zeta_{\mu \nu}\right)+L_{m}\left(\Psi, g_{\mu \nu}, \zeta_{\mu \nu}\right)\right) \sqrt{-g} d^{4} x
$$

In the above,

$$
L_{g}=-\frac{1}{2} \kappa_{g}^{2} R\left(g_{\mu \nu}\right)
$$

is the GR Lagrangian, $\kappa_{g}$ the Planck mass, and

$$
\Delta L_{g}=\Delta K\left(B_{\mu \nu}^{\lambda}, g_{\mu \nu}, \Sigma^{\mu}{ }_{\nu}\right)-V\left(\Sigma_{\nu}^{\mu}\right)
$$


is the additional gravity Lagrangian consisting of the potential $V$, containing the mass and self-interaction terms, and the quadratic kinetic term $\Delta K$. The latter is given by the sum, $\Delta K=\sum \kappa_{i} K_{i}$, of the partial contributions as follows:

$$
\begin{aligned}
& K_{1}=g^{\mu \nu} B_{\mu \kappa}^{\kappa} B_{\nu \lambda}^{\lambda}, \quad K_{2}=g_{\mu \nu} g^{\kappa \lambda} g^{\rho \sigma} B_{\kappa \lambda}^{\mu} B_{\rho \sigma}^{\nu}, \\
& K_{3}=g^{\mu \nu} B_{\mu \nu}^{\kappa} B_{\kappa \lambda}^{\lambda}, \quad K_{4}=g_{\mu \nu} g^{\kappa \lambda} g^{\rho \sigma} B_{\kappa \rho}^{\mu} B_{\lambda \sigma}^{\nu}, \\
& K_{5}=g^{\mu \nu} B_{\mu \kappa}^{\lambda} B_{\nu \lambda}^{\kappa},
\end{aligned}
$$

with the coefficients $\kappa_{i}$, generally, dependent on $\Sigma$.

\subsection{Linear approximation: physics content}

Choosing the flat backgrounds, putting $x^{\mu}=\delta_{\alpha}^{\mu} z^{\alpha}$, designating $Z^{\alpha}=\delta_{a}^{\alpha} Z^{a}$, and (with indices operated by $\eta_{\alpha \beta}$ ) decomposing the fields as

$$
\begin{aligned}
Z^{\alpha} & =z^{\alpha}+\zeta^{\alpha}, \\
g_{\alpha \beta} & =\eta_{\alpha \beta}+h_{\alpha \beta}, \\
\zeta_{\alpha \beta} & =\eta_{\alpha \beta}+\left(\partial_{\alpha} \zeta_{\beta}+\partial_{\beta} \zeta_{\alpha}\right), \\
\Sigma_{\alpha \beta} & =\eta_{\alpha \beta}-h_{\alpha \beta}+\left(\partial_{\alpha} \zeta_{\beta}+\partial_{\beta} \zeta_{\alpha}\right),
\end{aligned}
$$

one arrives in the linear approximation (LA) at the substitutions

$$
\begin{aligned}
\mathrm{LA}: \quad h_{\alpha \beta} \rightarrow \chi_{\alpha \beta}=h_{\alpha \beta}-\left(\partial_{\alpha} \zeta_{\beta}+\partial_{\beta} \zeta_{\alpha}\right), \\
h \equiv h_{\alpha}^{\alpha} \rightarrow \chi=h-2 \partial_{\alpha} \zeta^{\alpha} .
\end{aligned}
$$

This is nothing but the realization in LA of the SSB mechanizm for gravity. In the general case, GDiff gets completely broken/"hidden ", with all ten gravity components $\chi_{\alpha \beta}$ becoming physical and describing the scalar, vector and tensor gravitons. Nevertheless, depending on the particular choice for the Lagrangian parameters, there may be left some residual Diff symmetry to eliminate a part of the components (especially those corresponding to the putatively "dangerous "vector graviton). ${ }^{2}$ To illustrate, let us note the two following physically typical cases.

- Massive scalar graviton ${ }^{2} \sigma=\chi / 2$ at

$$
\Delta K=\kappa_{1} K_{1}=\kappa_{1} g^{\mu \nu} \partial_{\mu} \sigma \partial_{\nu} \sigma
$$

with $\kappa_{1}$, generally, dependent on $\sigma$, and $V=V(\sigma) \simeq m_{s}^{2} / 2 \sigma^{2}$ in addition to $L_{g}\left(\chi_{\alpha \beta}\right)$ of GR. Due to the residual transverse diffeomorphism (TDiff) symmetry

$$
\text { TDiff ; } \chi_{\alpha \beta} \rightarrow \chi_{\alpha \beta}+\partial_{\alpha} \varphi_{\beta}+\partial_{\beta} \varphi_{\alpha}, \quad \partial_{\gamma} \varphi^{\gamma}=0,
$$

transforming the trace and traceless part of $\chi_{\alpha \beta}$ separately as the proper irreducible representations, there is left the three physical components: one for the massive scalar graviton plus two for the massless tensor one, as in GR. ${ }^{\mathrm{h}}$

${ }^{\mathrm{h}}$ For the scalar graviton in the DM and DE contexts, see. ${ }^{3,4}$ At that, the results obtained in ${ }^{3}$ at a non-dynamical scalar density remain still valid at the dynamical $\zeta$. 
- Massive tensor graviton ${ }^{5-7} \gamma_{\alpha \beta}=\chi_{\alpha \beta} / 2$ at $\Delta K=0$ and $V=V(\Sigma)$ chosen properly to reduce in LA to the conventional Fiertz-Pauli potential $V_{\mathrm{FP}}\left(\chi_{\alpha \beta}\right)$. Under such a choice, there is left for the massive tensor graviton precisely five physical components, without ghosts.

A wide variety of the more sophisticated cases for SSB of GDiff may a priori be envisaged in QMG, with DCs beyond the two simplest realizations discussed above. All such cases can hardly be rejected just ab initio. To explore them is a challenge.

\section{Resume}

- QMG presents a bold and new type of the GR modification built on the mechanism of SSB for gravity. This is assured by a set of the (chart-wise reversible) quasi-affine dynamical coordinates, given by the scalar quartet, serving as the gravity counterpart of the Higgs fields.

- The mere admixture to metric of the scalar quartet is able to give rise to the extremely wide spectrum of the emergent gravity phenomena beyond GR, such as the massive tensor and scalar gravitons, the gravitational DM and/or DE, etc.

- Further theoretical and phenomenological study of QMG is needed to validate it (if any) as a candidate on the beyond GR theory of gravity. Accounting for the influence of vacuum, QMG as EFT may ultimately pave the way towards a more fundamental theory of gravity and space-time.

\section{References}

1. C. Patrignani et. al., Review of Particle Physics, Cin. Phys. C 40, 100001 (2016).

2. Y. F. Pirogov, Quartet-metric general relativity: scalar graviton, dark matter and dark energy, Eur. Phys. J. C 76, 215 (2016), arXiv:1511.04742 [gr-qc].

3. Y. F. Pirogov, Unimodular bimode gravity and the coherent scalar-graviton field as galaxy dark matter, Eur. Phys. J. C 72, 2017 (2012), arXiv:1111.1437 [gr-qc].

4. Y. F. Pirogov, Beyond LCDM per scalar graviton as the unified dark matter and dark energy, arXiv:1606.06150 [gr-qc].

5. A. H. Chamseddine and V. Muhanov, Higgs for graviton: simple and elegant solution, JHEP 1008:011 (2010), arXiv: 1002.3877 [hep-th].

6. I. Oda, Higgs mechanism for gravitons, Mod. Phys. Lett. A 25, 2411 (2010), arXiv: 1003.1437 [hep-th].

7. I. Oda, Remarks on Higgs mechanism for gravitons, Phys. Lett. B 690, 322 (2010), arXiv: 1004.3078 [hep-th].

8. E. I. Guendelman and A. B. Kaganovich, The principle of non-gravitating vacuum energy and some of its consequences, Phys. Rev. D 53, 7020 (1996), arXiv:gr-qc/ 9605026.

9. E. I. Guendelman and A. B. Kaganovich, Physical consequences of a theory with dynamical volume element, arXiv:0811.0793 [gr-qc] . 